\title{
Lipid accumulation facilitates mitotic slippage-induced adaptation to anti-mitotic drug treatment
}

\author{
Alex Wong ${ }^{1,2}$, Sixun Chen ${ }^{1,3}$, Lay Kien Yang ${ }^{4}$, Yoganathan Kanagasundaram ${ }^{4}$ and Karen Crasta ${ }^{1,3,5}$
}

\begin{abstract}
Aberrant lipid accumulation is a hallmark of cancer known to contribute to its aggressiveness and malignancy. Emerging studies have demonstrated context-dependent changes in lipid metabolism during chemotherapy. However, there is little known regarding the mechanisms linking lipid metabolism to chemotherapy-induced cell fates. Here, we describe lipid accumulation in cells following antimitotic drug treatment. Cells arrested in mitosis, as well as cells that escaped mitotic arrest and underwent mitotic slippage, showed elevated cytoplasmic lipid droplets. Interestingly, we found that TOFA, a lipid biosynthesis inhibitor that targets acetyl-CoA carboxylase (ACC) and blocks lipid accumulation, promoted early slippage, reduced cellular stress and enhanced survival of antimitotic-treated cells. Our work previously revealed that cells that survive after mitotic slippage can become senescent and confer protumourigenic effects through paracrine signalling. Modulating lipid biosynthesis in cells post slippage by TOFA amplified their inflammatory secretion profiles and accelerated the development of tumourigenic behaviour, particularly cell migration and invasion, in a paracrine-dependent manner. In contrast to TOFA, inhibition of lipid accumulation by C75, a drug targeting fatty acid synthase (FASN), significantly reduced the production of protumourigenic factors and associated phenotypic effects. This suggests that discrete lipid biosynthesis pathways could contribute differentially to the regulation of pro-tumourigenic inflammation. The divergent effects of TOFA and C75 may be attributed to the opposing regulation of Malonyl-CoA, an intermediate in fatty acid synthesis that serves as a mediator of fatty acid oxidation. Taken together, our data reveal a previously unappreciated role for lipid accumulation in the cellular adaptation to antimitotic drug treatment. Targeting lipid biosynthesis in cells post slippage may reprogramme its secretory profile such that it not only negates tumour-promoting effects, but may also promote antitumour inflammation for clearance of post-slippage senescent cells.
\end{abstract}

\section{Introduction}

Antimitotic drugs, such as paclitaxel and vinblastine, are often used as first-line therapy against a broad range of cancers $^{1,2}$. By targeting microtubule dynamics, these drugs affect cell proliferation culminating in a mitotic arrest and eventually mitotic cell death. However, cells

\footnotetext{
Correspondence: Karen Crasta (kccrasta@ntu.edu.sg)

${ }^{1}$ Lee Kong Chian School of Medicine, Nanyang Technological University,

Singapore, Singapore

${ }^{2}$ Institute for Health Technologies, Interdisciplinary Graduate School, Nanyang

Technological University, Singapore, Singapore

Full list of author information is available at the end of the article.

Edited by I. Amelio
}

could also take an alternative cell fate route known as mitotic slippage, a process where cells exit mitosis and enter interphase without going through proper chromosome segregation and cytokinesis ${ }^{3}$. As a result, cells post slippage tend to be tetraploid and multinucleated. Previous studies have described various cell fates post slippage including: (1) apoptosis, (2) cell cycle arrest that culminates in senescence and (3) proliferation as genomically unstable cells ${ }^{4}$.

While several mechanistic studies have alluded to cell death post slippage ${ }^{5,6}$, there has been little describing molecular pathways leading to cell cycle arrest and the

\section{(c) 2018 The Author(s).}

\footnotetext{
(c) (i) Open Access This article is licensed under a Creative Commons Attribution 4.0 International License, which permits use, sharing, adaptation, distribution and reproduction cc) in any medium or format, as long as you give appropriate credit to the original author(s) and the source, provide a link to the Creative Commons license, and indicate if changes were made. The images or other third party material in this article are included in the article's Creative Commons license, unless indicated otherwise in a credit line to the material. If material is not included in the article's Creative Commons license and your intended use is not permitted by statutory regulation or exceeds the permitted use, you will need to obtain permission directly from the copyright holder. To view a copy of this license, visit http://creativecommons.org/licenses/by/4.0/.
} 
ensuing senescence post slippage. We have previously shown that the senescence-associated secretory phenotype (SASP) factors ${ }^{7}$, consisting of various cytokines, chemokines and growth factors released by post-slippage senescent cells, promote tumourigenic behaviour in neighbouring cells ${ }^{8}$. Persistence of cells post slippage may undermine the effectiveness of antimitotic drugs and ultimately contribute to the development of tumour recurrence and chemoresistance. Hence, it is crucial to gain better mechanistic understanding of the senescent cell fate post slippage for enhanced therapeutic strategies involving the elimination of senescent cells or its associated pro-tumourigenic effects post slippage following antimitotic therapy.

Enhanced lipid biosynthesis is a characteristic feature of cancers. Indeed, aberrant lipid accumulation in cancer cells has emerged as a possible diagnostic and therapeutic target ${ }^{9}$. In cancer cells, the supply of cellular fatty acids is highly dependent on the de novo fatty acid synthesis ${ }^{10}$. This involves two key enzymes, acetyl-CoA carboxylase (ACC) and fatty acid synthase (FASN). ACC carboxylates acetyl-Co to form malonyl-CoA. The malonyl-CoA is further converted to long-chain fatty acids by FASN. Acyl-CoA synthetase then coverts fatty acid to acyl-CoA.

Chemotherapeutic drugs doxorubicin and 5fluorouracil that are used in the treatment of human colorectal and breast cancer cells have previously been reported to induce the accumulation of cytoplasmic lipid droplets (LDs) ${ }^{11-13}$. Additionally, LD induction during apoptosis in murine lymphoma cells treated with etoposide has been shown to result from inhibition of mitochondrial fatty acid oxidation, in which fatty acids are directed towards the de novo fatty acid synthesis ${ }^{14}$. A similar mechanism governing LD accumulation was described in neuroblastoma cells treated with a c-Myc/ Max inhibitor ${ }^{15}$. Ceramide metabolism has also been implicated as a key regulator of sensitivity to paclitaxel and other chemotherapeutic drugs ${ }^{16}$. Importantly, in addition to apoptotic cells, a role for LD accumulation has also been observed in senescent cells as well. Senescent cells have been shown to contain LDs that are more numerous and larger in size than their proliferating counterparts ${ }^{17}$. Murine melanoma cells incubated with delipidised media containing specific lipids such as ceramide and triglyceride significantly increased cellular senescence, suggesting that modulated lipid metabolism could contribute to the onset of senescence ${ }^{17}$.

Treatment with paclitaxel, the most commonly utilised antimitotic drug in the clinics, has been shown to induce LD accumulation following treatment ${ }^{18}$. Increased LD formation has been detected in both mitotically arrested and apoptotic cells, with the latter showing relatively higher accumulation. However, it is unknown if lipid biosynthesis influences cell fate following antimitotic drug treatment, particularly implications for mitotic slippage. Here, we describe a role for aberrant lipid metabolism during both mitotic arrest and post slippage following treatment with the antimitotic drug Nocodazole (Noc). We show that 5-(tetradecyloxy)-2-fuoric acid (TOFA), a lipid biosynthesis inhibitor that targets ACC and blocks lipid accumulation, promoted early slippage. The ensuing cellular stress was reduced, which in turn increased survival of antimitotic-treated cells. TOFA treatment also increased production of secretory factors that potentiated tumourigenic phenotypes, such as enhanced migration and invasion post slippage. Notably, senescence induction per se was not perturbed by lipid inhibition. Intriguingly, unlike TOFA-induced lipid inhibition, inhibition by C75 that targets FASN (an enzyme directly downstream of ACC) drastically reduced the production of inflammatory secretory factors in post-slippage cells via modulation of the NF- $\mathrm{kB}$ (nuclear factor kappa-light-chain-enhancer of activated B cells) pathway. This conundrum points to specific and differential inhibition of the de novo fatty acid synthesis pathway via inhibitors used. Therefore, it is plausible that discrete lipid biosynthetic pathways contribute differentially to the regulation of pro-tumourigenic inflammation post slippage. Taken together, our results reveal a previously unappreciated consequence of lipid accumulation in the regulation of mitotic cell fate, particularly mitotic slippage, and its associated protumourigenic inflammation in response to treatment with antimitotic drugs.

\section{Results}

Antimitotic drugs induce lipid accumulation during mitotic arrest and after slippage

To interrogate the effects of antimitotic drugs in modulating lipid metabolism, we first treated mitotic slippageprone osteosarcoma U2OS cells with Noc and assessed lipid accumulation in these cells. Consistent with our previous observations ${ }^{8}$, more than $80 \%$ of U2OS cells underwent mitotic slippage and displayed multinucleation, following Noc treatment for $48 \mathrm{~h}$ (Supplementary Fig. S1a). This was further confirmed by western blot profiles showing time-dependent degradation of mitotic markers cyclin B1, decreased phosphorylation of histone- $\mathrm{H} 3$ (pH-H3) and decreased levels of spindle assembly checkpoint marker BubR1 (Supplementary Fig. S1b). To assess lipid accumulation post slippage, U2OS cells were treated with Noc for $48 \mathrm{~h}$ and subsequently stained with Oil Red $\mathrm{O}$, a dye that stains neutral triglycerides and LDs. An increase in cytoplasmic LDs was observed in U2OS cells post slippage compared to dimethyl sulfoxide (DMSO)-treated control cells (Fig. 1a). Triascin C (TC), an inhibitor of long fatty acyl-CoA synthetase (an essential enzyme for lipid droplet biosynthesis), significantly reduced LD accumulation in Noc- 


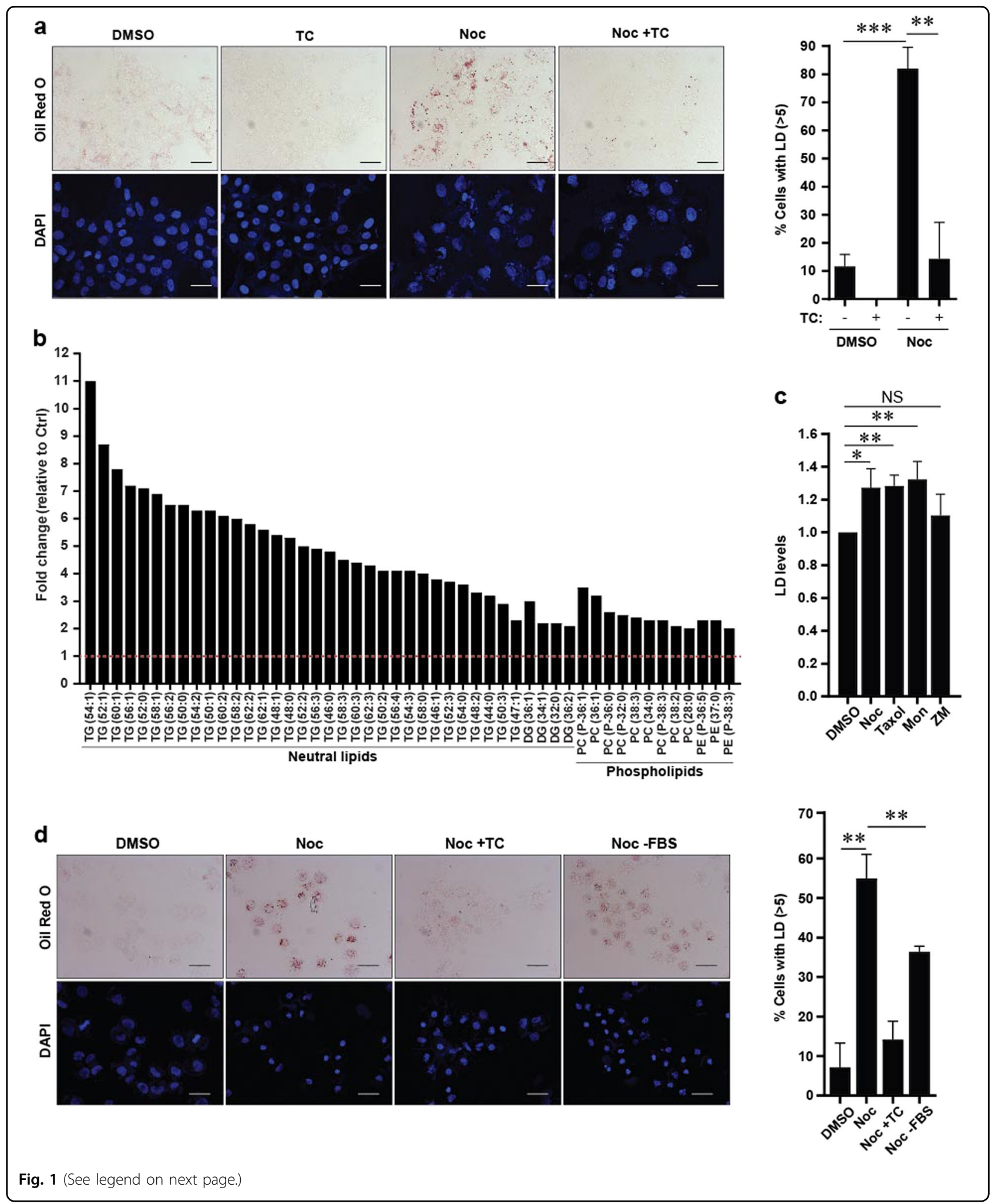

treated cells post slippage, confirming a bona fide lipid accumulation (Fig. 1a). LD accumulation was also observed in HCT116 human colon carcinoma and MDAMB-231 breast adenocarcinoma cell lines post slippage following treatment with Noc for $48 \mathrm{~h}$ (Supplementary Fig. S2a, b).

To further validate lipid accumulation in cells post slippage, we performed lipidomic analyses of DMSO- 
(see figure on previous page)

Fig. 1 Lipid accumulation is induced in mitotically arrested and cells post slippage. a Left: Representative images of U2OS cells treated with DMSO or Noc for $48 \mathrm{~h}$ in the presence or absence of Triascin C (TC). Cells were stained with Oil Red O. Scale bar: 50 um. Right: Quantification of cells with $>5$ LDs from (a). Data are mean \pm s.d. of 3 independent experiments. $\mathbf{b} \cup 2 \mathrm{OS}$ cells treated with Noc for $48 \mathrm{~h}$ were collected for lipidomic analysis. Each class of lipids was normalised with respective internal standards. Lipids identified were matched by Lipid Maps and confirmed with MS/MS. Lipid profiles shown are greater than 2-fold change $(p>0.05)$ with respect to DMSO-treated (Ctrl) cells. c Flow cytometric analysis of U2OS cells treated with different classes of antimitotic drugs for $48 \mathrm{~h}$ and analysed using BODIPY 493/503 staining. LD levels represent the mean fluorescence intensity values of the total cell population for each sample. Data are mean \pm s.d. of 3 independent experiments. $\mathbf{d}$ Left: Representative images of U2OS cells treated with the indicated drugs or inhibitors for $24 \mathrm{~h}$ and stained for Oil Red O (red) and DAPI (blue). Scale bar: $50 \mu \mathrm{m}$. Right:

Quantification of cells with $>5$ LDs from (d). Data are mean \pm s.d. of 3 independent experiments; ${ }^{*} p<0.05,{ }^{* *} p<0.01,{ }^{* * *} p<0.001$ and NS nonsignificance by Student's t-test

treated and $48 \mathrm{~h}$ Noc-treated U2OS cells using mass spectrometry. Lipidomic profiles normalised to respective internal standards revealed a significant upregulation of lipid metabolites, particularly the lipid species triacylglycerol (TAGs), in the Noc-treated samples compared to control cells (Fig. 1b). TAGs are neutral lipid species that are well defined as the major composition found in LDs ${ }^{19}$. To test whether LD accumulation occurs under other antimitotic drug conditions that can also induce mitotic slippage after $48 \mathrm{~h}$ of treatments, we assayed for neutral lipid species using the fluorescent neutral lipid dye 4,4difluoro-1,3,5,7,8-pentamethyl-4-bora-3a,4a-diaza-s-indacene (BODIPY 493/503). Flow cytometric detection of BODIPY 493/503 confirmed increased LD accumulation in cells post slippage induced by various classes of antimitotic drugs such as microtubule-stabilising Taxol, mitotic kinesin Eg5 inhibitor Monastrol and Aurora-B kinase inhibitor ZM447439 compared to DMSO-treated cells (Fig. 1c). ZM447439 overcomes the spindle assembly checkpoint, leading to an earlier exit from mitotic arrest and entry into mitotic slippage. It was observed that LD formation in ZM447439-treated cells was not significantly increased compared to control (Fig. 1c). This led us to ask whether arrest in mitosis was necessary for lipid accumulation post slippage. To this end, we treated cells with Noc for $24 \mathrm{~h}$ to enforce a prolonged mitotic arrest. We observed that approximately $56 \%$ of mitotically arrested cells were observed to contain LDs compared to DMSOtreated cells (8\%) (Fig. 1d). In addition, we observed a modest reduction in the percentage of LD-abundant mitotically arrested cells when cultured in serum-depleted media ( $0 \%$ foetal bovine serum (FBS)) (Fig. 1d), indicating that exogenous lipid uptake may contribute to the elevated lipid content. Overall, our findings suggest that antimitotic drug-induced lipid accumulation first accumulates during mitotic arrest, and consequently following mitotic slippage.

\section{Fatty acid uptake contributes to lipid accumulation in cells post slippage}

The accumulation of lipids induced by anticancer drugs such as bevacizumab was shown to be due to an increased uptake of fatty acids (FAs) from the surrounding environment ${ }^{20}$. Since a slight reduction in LDs was observed in serum-depleted cells, we hypothesised that extracellular FA may contribute to lipid accumulation in our context as well. To assess the effect of Noc treatment on FA uptake in U2OS cells, we stimulated FA uptake by means of serum starvation where FBS addition into the culture media was omitted. Significant upregulation in the rate of FA uptake was observed in U2OS cells treated with Noc for $48 \mathrm{~h}$ compared to the DMSO-treated control (Fig. 2a, b). In support of this, microarray analysis of gene expression in Noc-induced post-slippage cells revealed a significant enrichment of fatty acid-binding protein 4 (FABP4) messenger RNA (mRNA) as compared to DMSO-treated cells (accession number: GSE114515) (Supplementary Table 1). Fatty acid-binding proteins (FABPs) belong to a family of cytoplasmic proteins known to be involved in FA uptake and transport ${ }^{20,21}$. We assessed the expression of FABPs by mRNA expression analysis which confirmed that FABP4 is significantly elevated in U2OS cells post slippage compared to control cells (Fig. 2c). To determine whether FABP4 could promote Noc-induced LD accumulation, we co-treated cells with Noc and a small molecule inhibitor of FABP4 (F4I), BMS309403. Inhibition of FABP4 led to a modest reduction in LD level in Noc-treated cells (Fig. 2d). Our findings suggest that FABP4 could mediate enhanced FA uptake that may partially contribute to LD accumulation in cells post slippage.

\section{Blocking acetyl-CoA carboxylase suppresses cellular stress and promotes survival of cells post slippage}

It remains unclear as to whether LD accumulation upon antimitotic drug treatment is associated with cell survival or death. To ascertain this, we evaluated the cytotoxicity of various classes of antimitotic drugs described above (ZM447439, Monastrol, Nocodazole and Taxol) in U2OS cells. Relative cell viability was correlated to their respective cellular LD levels. Intriguingly, an inverse correlation between cell viability post treatment and LD levels was observed, suggesting that LD accumulation is associated with cell death (Fig. 3a). To further characterise the link between LD accumulation and antimitoticinduced cell death, we blocked lipid accumulation with 
a
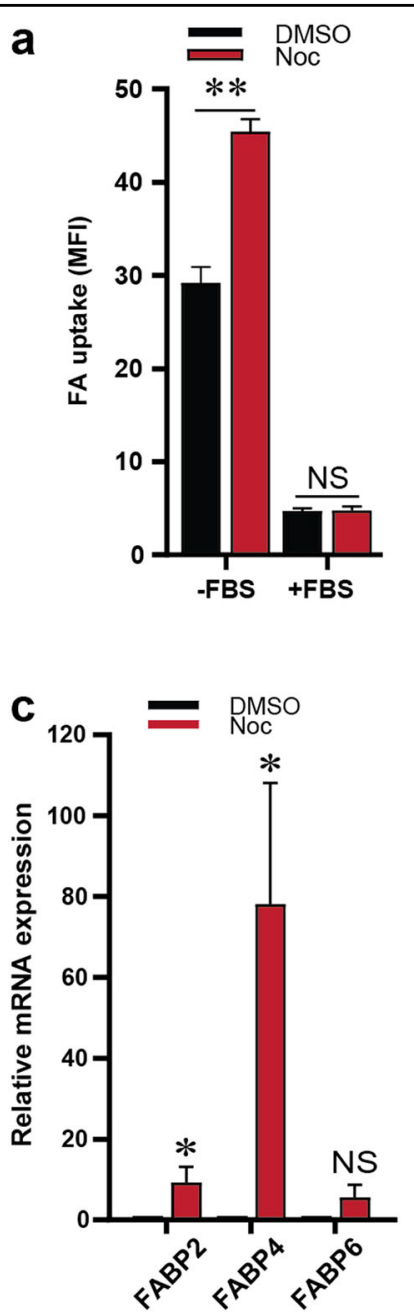

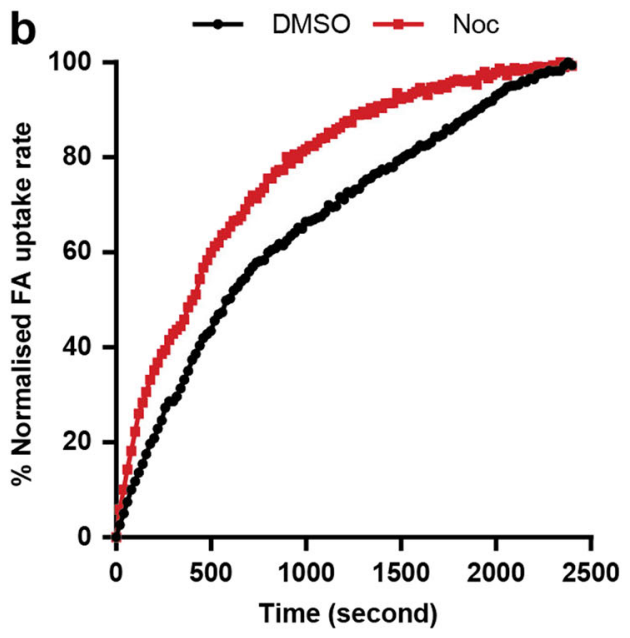

d

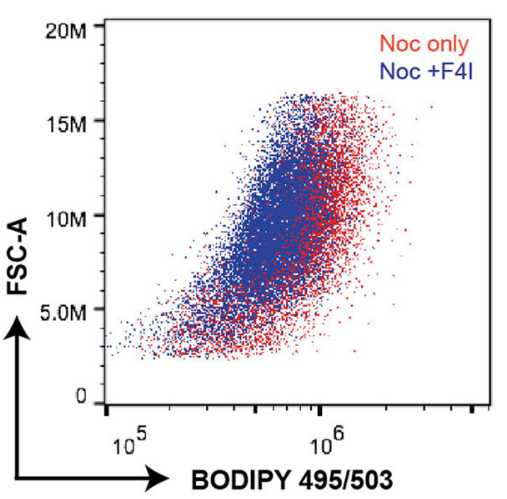

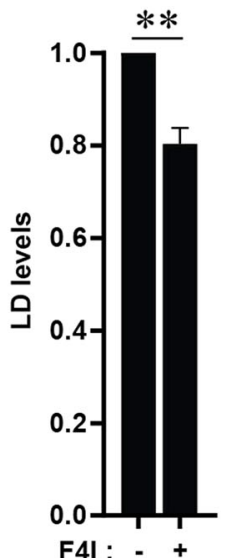

Fig. 2 Enhanced fatty acid uptake contributes to LD accumulation. a FA uptake in U2OS cells treated with DMSO or Noc for 48 h. Serum starvation (SS) was performed after drug treatment to stimulate FA uptake. Negative control of FA uptake assay: normal serum (10\% FBS) condition. Measurement of FA uptake in cells was quantified by mean fluorescence intensity (MFI). Data are mean \pm s.d. of 3 independent experiments. b Representative plot of fatty acid uptake kinetics performed as per (a). c Synchronised U2OS were treated with DMSO or Noc for $48 \mathrm{~h}$ and analysed for FABP mRNA expression. $\mathbf{d}$ Flow cytometric analysis of U2OS cells treated with Noc alone or in combination with different concentrations of FABP4 inhibitor (F4I). Cells were stained for LDs using BODIPY 493/503. Data are mean \pm s.d. of 3 independent experiments; ${ }^{*} p<0.05,{ }^{* *} p<0.01$ and NS nonsignificance by Student's t-test

a pharmacological inhibitor of lipid biosynthesis, TOFA. TOFA inhibits the rate-limiting enzyme of fatty acid biosynthesis pathways ACC which converts acetyl-CoA to malonyl-CoA (Supplementary Fig. 3a). Our results indicate that TOFA prominently reduced LD accumulation in Noc-induced cells post slippage (Supplementary Fig. 3b). Co-treatment of Noc and TOFA was able to further augment reduction of LDs and promote short- and longterm survival of cells post slippage (Fig. 3b-d). It is known that cells post slippage can acquire extensive DNA damage from prolonged mitotic arrest and multinucleation $^{5,22,23}$. Therefore, TOFA treatment might alleviate antimitotic drug-induced stress response and promote cellular survival. Indeed, we observed that TOFA-treated cells post slippage displayed dramatic reduction in DNA damage ( $\gamma$-H2AX levels) and reactive oxygen species (ROS) levels as compared to the LD-laden cells (Fig. 3e, f).

Previous studies have shown that duration of mitotic arrest dictates the extent of multinucleation and DNA damage post slippage ${ }^{22}$. We hypothesised that lipid inhibition by TOFA may reduce the duration of mitotic arrest and promote early slippage upon Noc treatment. Our results demonstrate an increased degradation of cyclin B1, a trigger of mitotic exit ${ }^{24}$ and reduction in phosphorylated histone H3 levels when cells were co-treated with Noc and TOFA (Fig. 4a, b), suggesting earlier slippage. Consistent with this, we observed a modest increase in the percentage of multinucleated cells co-treated with TOFA at an early time point $t=16 \mathrm{~h}$, compared to cells treated 

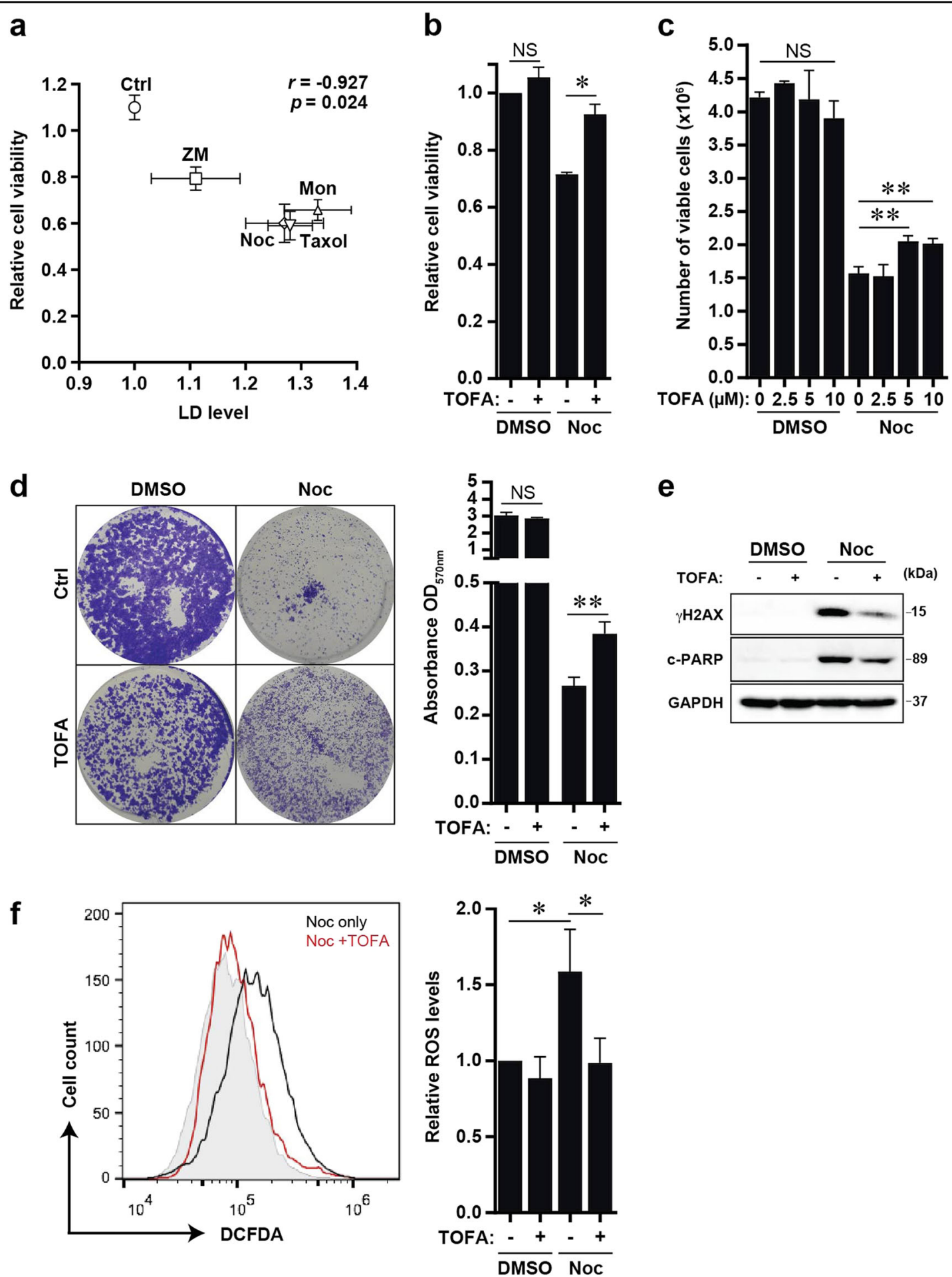

Fig. 3 Lipid biosynthesis inhibitor TOFA suppresses cellular stress and promotes the survival of cells post slippage. a Cell viability and LD levels in U2OS cells after $48 \mathrm{~h}$ of treatment with respective antimitotic drugs. Cell viability was compared to respective LD levels and Pearson's correlation coefficients were determined. b Synchronised U2OS cells were treated with DMSO or Noc in the presence or absence of TOFA for $48 \mathrm{~h}$, replated and grown for $24 \mathrm{~h}$ before cell viability assay was performed. Data are mean \pm s.d. of 2 independent experiments. c Synchronised U2OS cells were treated with DMSO or Noc alone or in combination with TOFA for $48 \mathrm{~h}$ before quantifying the number of viable cells. Data are mean \pm s.d. of 3 independent experiments. $\mathbf{d}$ Left: Longterm clonogenic assay of U2OS cells treated with DMSO or Noc in the presence or absence of TOFA. Cells were treated with respective drugs for $48 \mathrm{~h}$, cultured in fresh media for another 5 days before crystal violet staining. Right: Plot shows the quantification of crystal violet staining. Data are mean \pm s.d. of 3 independent experiments. e Western blot of U2OS cells treated with respective drugs for $48 \mathrm{~h}$. $\mathbf{f}$ Left: Flow cytometry analysis of synchronised U2OS cells treated with respective drugs for $48 \mathrm{~h}$, followed by DCFDA staining. Right: Quantification of ROS level in U2OS cells treated with respective drugs for $48 \mathrm{~h}$. Data are mean \pm s.d. of 3 independent experiments; ${ }^{*} p<0.05,{ }^{* *} p<0.01$ and NS non-significance by Student's $t$-test 


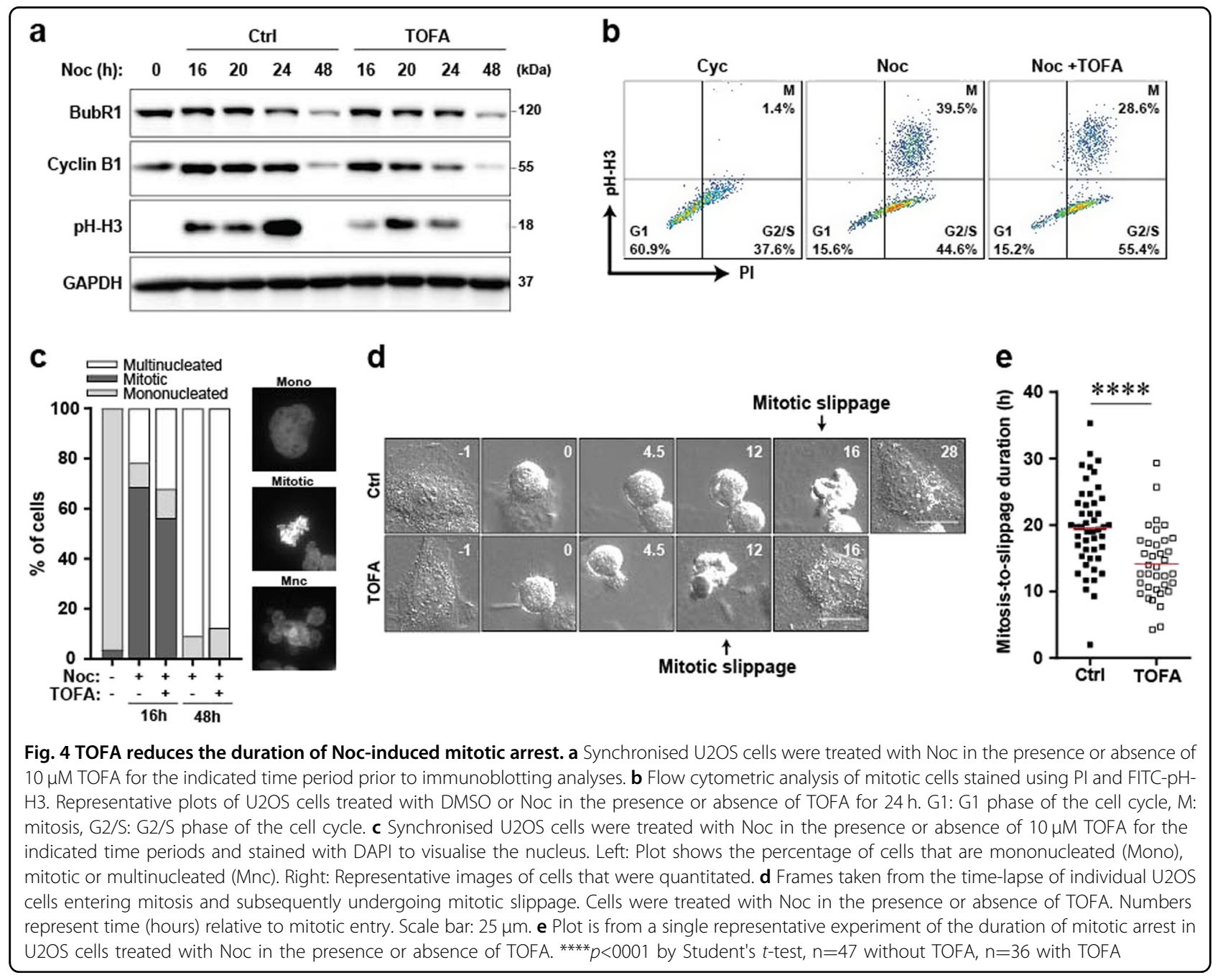

with Noc alone (Fig. 4c). To confirm this, we used live-cell imaging to discern the duration of mitotic arrest by measuring the time interval from cell rounding to mitotic slippage. A significant reduction in the duration of mitotic arrest was observed when cells were co-treated with TOFA and Noc (Fig. 4d, e). All the above suggest that blocking ACC in the lipid biosynthesis pathway with TOFA reduced the duration of Noc-induced mitotic arrest, which in turn decreased DNA damage and ROS levels in cells post slippage, possibly leading to their survival.

\section{Lipid biosynthesis differentially regulates post-slippage production of inflammatory factors and tumourigenic effects}

We have previously shown that cells post slippage enter senescence, exhibit SASP and secrete pro-inflammatory factors such as interleukin (IL)-6, IL-8 and IL-1 $\beta$ which promote paracrine pro-tumourigenic effects such as migration, invasion and angiogenesis ${ }^{8}$. With regards to lipid metabolism, it has recently been revealed that therapy-induced senescent cells promote the build-up of lipids that potentially contribute to senescence induction ${ }^{17}$. To determine whether lipid biosynthesis regulates senescence following mitotic slippage, we treated U2OS cells with either Noc alone for $72 \mathrm{~h}$ or in combination with TOFA, and stained for the senescence-associated beta-galactosidase (SA- $\beta$-gal). Surprisingly, SA- $\beta$-gal staining revealed that there was no discernible difference in senescence establishment following mitotic slippage, with or without treatment with TOFA (Supplementary Fig. 4a). In addition, no discernable changes were observed in the expression of the senescence-associated Lamin B1 and p21 between control and TOFA-treated cells post slippage (Supplementary Fig. 4b), confirming that TOFA treatment did not affect post-slippage senescence induction. Interestingly, western blots also revealed augmented expression of the dominant cytokines detected post slippage as previously reported ${ }^{8}$, IL- 8 and IL- $1 \beta$, upon co-treatment of Noc and TOFA 
(Supplementary Fig. 4b). To exclude the possibility that the observed cytokine induction by TOFA treatment was due to early mitotic slippage, we subjected cells to $48 \mathrm{~h}$ of Noc treatment to allow them to enter a "post-slippage state", followed by $24 \mathrm{~h}$ of treatment with TOFA. The conditioned media collected were analysed for secretion of pro-tumourigenic factors using enzyme-linked immunosorbent assay (ELISA)-based cytokine profiling (Fig. 5a). Consistent with our previous findings ${ }^{8}$, Noc-only treated cells showed increased secretion of several cytokines and chemokines such as IL-6, IL- 8 and IL- $1 \beta$ (Fig. 5b). Lipid inhibition via treatment with TOFA in cells post slippage was observed to further augment secretion of these factors compared to control cells (Fig. 5b). Interestingly, increased expression of IL-8 and IL-1 $\beta$ correlated with a dose-dependent increase of TOFA, further supporting the notion that lipid inhibition increases inflammatory factors post slippage (Fig. 5c). Treatment of TOFA alone in U2OS cells did not induce the expression of IL- 8 and IL- $1 \beta$, suggesting that TOFA specifically regulates pro-inflammatory factors post slippage (Fig. 5c).

To assess whether TOFA affects the migratory and invasive properties induced by the factors secreted, we collected conditioned media (CM) from control or TOFA-treated cells post slippage for use in the scratch wound healing and transwell invasion assays. As shown in Fig. 5d, CM obtained from TOFA-treated cells post slippage increased the rate of wound closure compared to control cells, suggesting increased migration. Likewise, the transwell assay showed increased invasion of the bottom of the transwell chamber by cells incubated with CM from TOFA-treated post-slippage cells, suggesting increased invasion compared to controls (Fig. 5e). Overall,

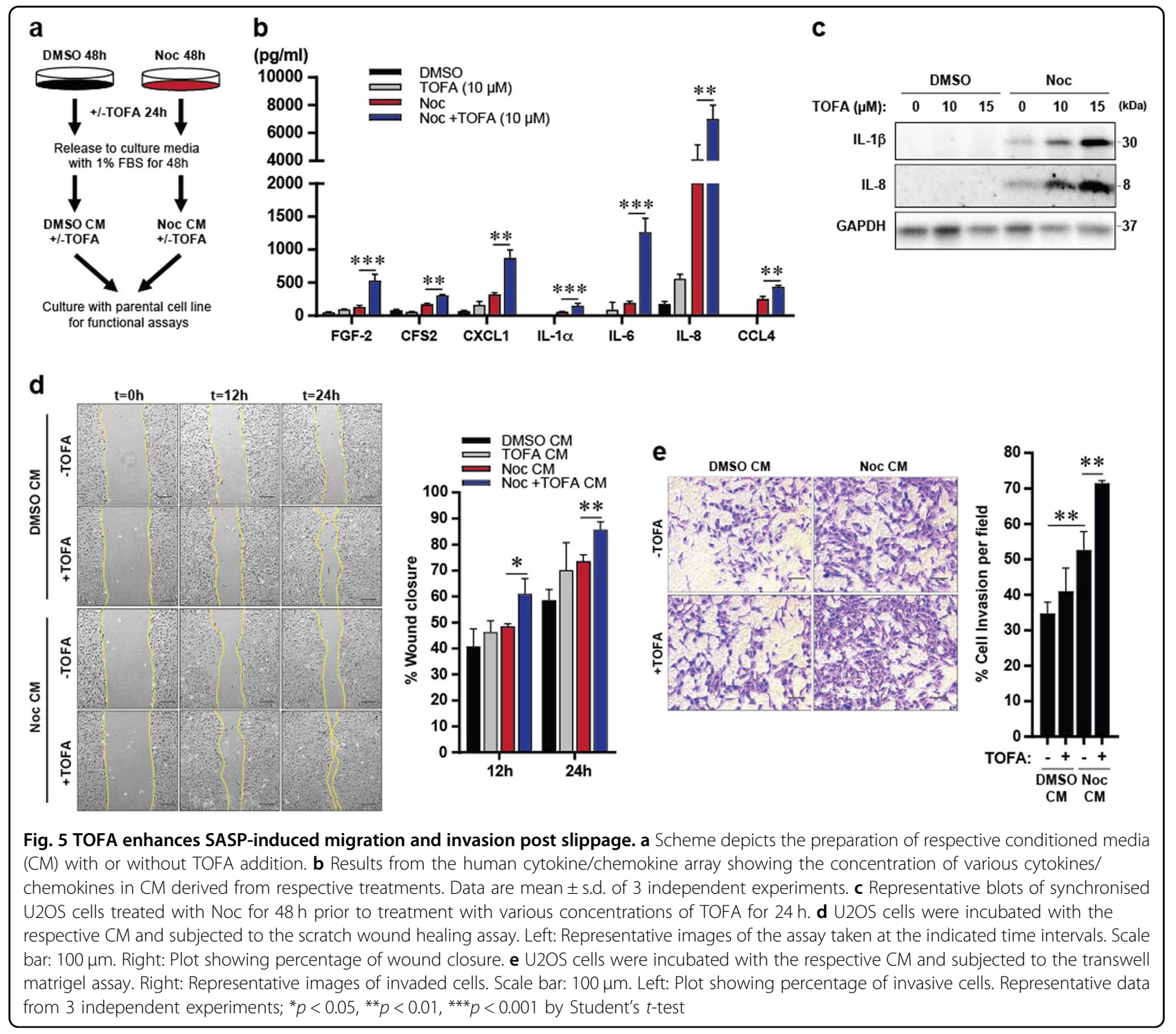


our results suggest that lipid inhibition by TOFA increased tumourigenic effects.

To further confirm the effect of lipid inhibition on inflammatory factors post slippage, we treated Nocinduced post-slippage cells with another inhibitor of lipid biosynthesis, $\mathrm{C75}$, and analysed the expression and secretory profiles of several cytokines and chemokines. C75 inhibits FASN that is directly downstream of ACC (Supplementary Fig. 3a) ${ }^{25}$. Surprisingly, contrary to TOFA, co-treatment with C75 suppressed the secretion of several cytokines and chemokines such as IL-6, IL-8 and IL-1 $\beta$ (Fig. 6a, b). This finding was further supported by co-treatment with Orlistat, another inhibitor of FASN, that also suppressed the expression of IL- 8 and IL- $1 \beta$
(Supplementary Fig. 5). In addition, CM derived from post-slippage cells co-treated with $\mathrm{C} 75$ attenuated the migratory and invasive capabilities compared to control (Fig. 6c, d). Our findings suggest that FASN inhibition by C75 could present a strategy to prevent tumourigenic effects following mitotic slippage. Our data suggest that targeting the fatty acid biosynthesis pathway at different nodal points may differentially regulate the production of inflammatory factors controlling tumourigenic action.

\section{FASN inhibition suppresses inflammatory cytokines by inhibiting NF-KB activity}

The production of pro-tumourigenic cytokines such as IL-1, IL- 6 and IL- 8 are known to be induced by the NF- $\mathrm{kB}$

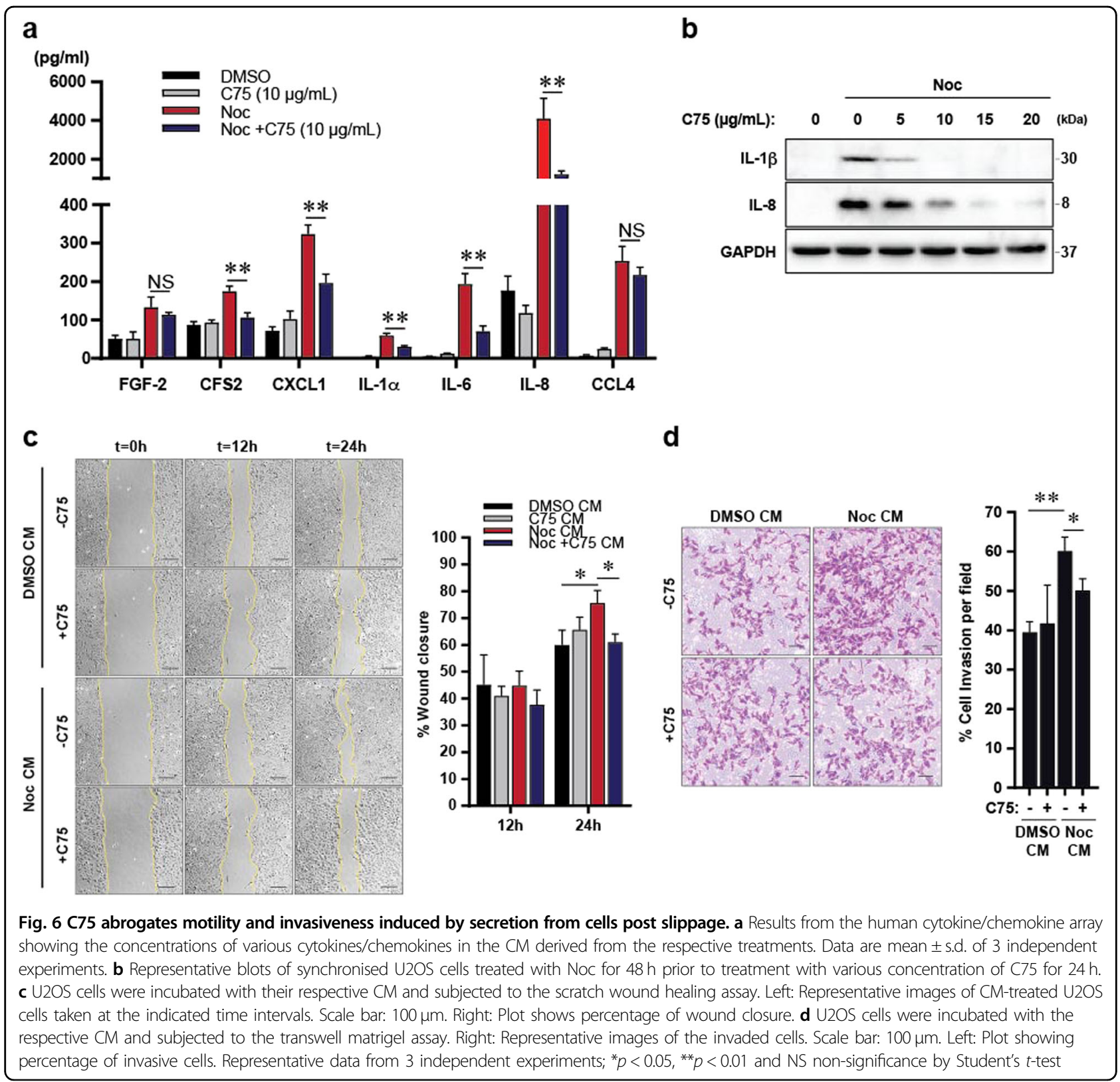


family of transcriptional factors ${ }^{26}$. Previous reports have also described activation of NF-кB-dependent inflammatory response upon paclitaxel treatment in multiple cancer cells ${ }^{27-29}$. Hence, one could predict that the suppression of pro-tumourigenic inflammation by FASN inhibitor C75 in cells following mitotic slippage could be due to the suppression of NF- $\mathrm{KB}$ activation. To test this, cells were co-treated with Noc and increasing concentrations of the NF- $\kappa$ B inhibitor Bay 11-7082. Results indicate that NF- $\mathrm{kB}$ inhibition resulted in the dosedependent reduction of IL- 8 and IL- $1 \beta$ expression, affirming that cytokine production upon Noc treatment is mediated through NF-kB pathway (Fig. 7a). Western blotting analysis revealed enhanced phosphorylation of $\mathrm{I} \kappa \mathrm{B} \alpha$ and $\mathrm{p} 65$, suggesting that the NF- $\mathrm{KB}$ pathway is activated in cells post slippage (Fig. 7b). Interestingly, treatment of cells post slippage with the C75 inhibitor

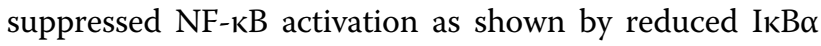

and p65 phosphorylation compared to DMSO-treated cells (Fig. 7c). This was further confirmed by the modest reduction of nuclear p65 in C75-treated cells compared to control (Fig. 7d). These results suggest that lipid inhibition by $\mathrm{C} 75$ suppressed the expression of inflammatory factors via NF- $\mathrm{kB}$ inactivation and subsequently perturbed the pro-tumorigenic effects induced by postslippage cells.

\section{Discussion}

Although many anticancer drug treatments induce lipid accumulation in cancer cells ${ }^{11-13,15,17,18}$, the consequences of this phenomenon in affecting the efficacy of anticancer drugs remain largely unknown. In this study, we explored the role of lipid metabolism in regulating cell fate upon treatment with antimitotic drugs. We had previously proposed mitotic slippage-induced senescence and SASP following antimitotic drug treatment to be an

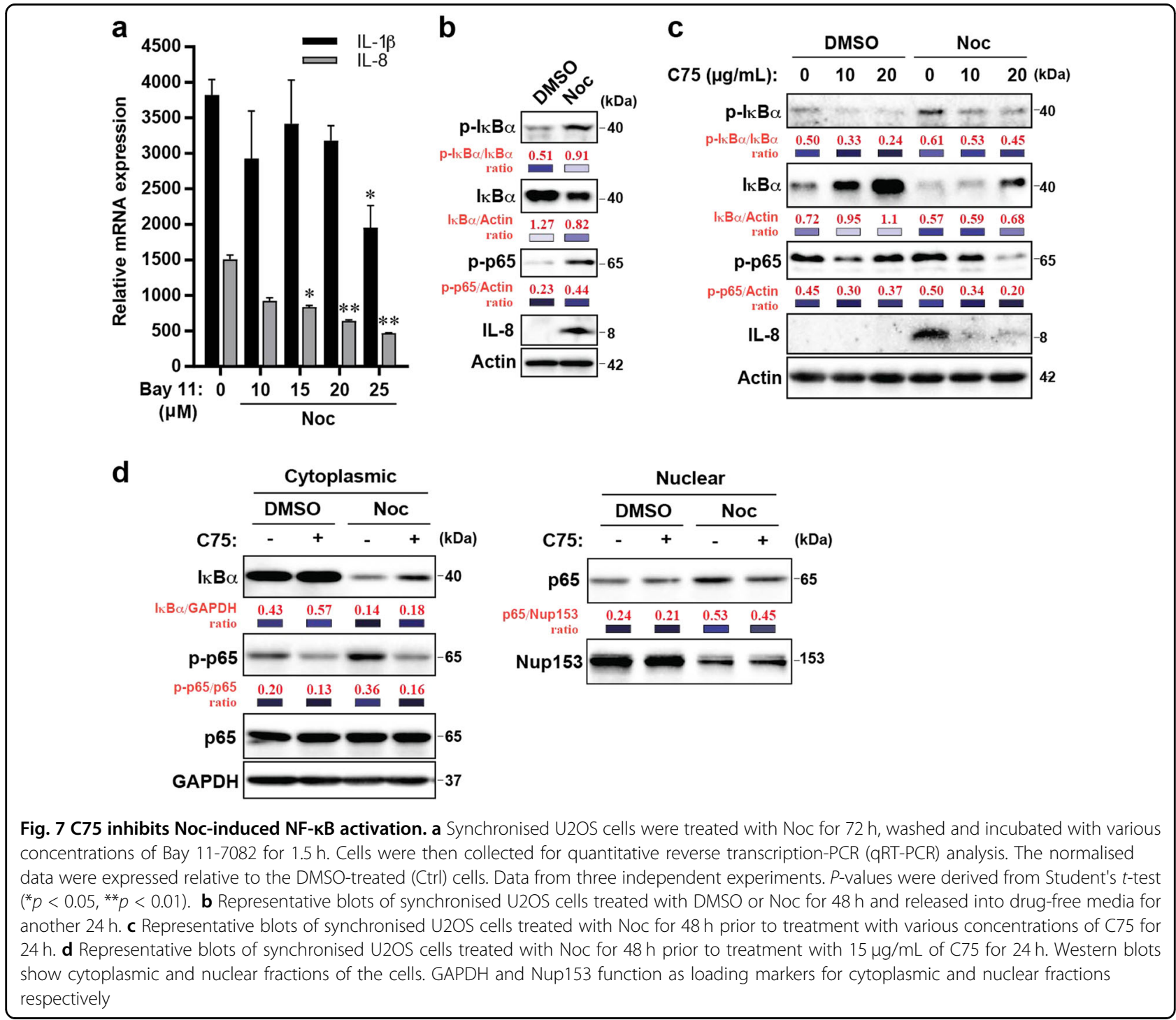


important determinant of cancer cell survival ${ }^{8}$. The findings presented here demonstrate pharmacological inhibitor-induced changes in lipid biosynthesis in cells treated with Noc and their implications for tumourpromoting effects of cells post slippage (Fig. 8). Intriguingly, we found that inhibition of lipid accumulation could promote early mitotic slippage and contribute to the survival of cells post slippage. Although the incidence of senescence per se was not affected, lipid inhibition could modulate production of secretory factors (SASP) post slippage in a NF-kB-dependent manner, contingent on the lipid biosynthetic pathway invoked. Our study highlights the potential of targeting lipid biosynthesis in cells post slippage to reprogramme its secretory profile in a manner that could not only negate tumour-promoting effects, but may also promote anti-tumour inflammation for clearance of tumour cells.

Lipid accumulation upon chemotherapy can be triggered by several mechanisms such as enhanced lipogenesis, increased uptake of lipids and inhibition of FA oxidation $^{30}$. Our study demonstrated that the accumulation of lipid content in cells post slippage was partly contributed by an increase in FABP4-mediated FA uptake. FABP4 is known to be upregulated in many aggressive cancers as well as recurrent tumours to facilitate the uptake and transport of extracellular FA from adipocytes to mitochondria for $\beta$-oxidation ${ }^{31-33}$. More work will have to be done to delineate the role of FABP4 in mediating post-slippage cell fate. Lipid accumulation upon antimitotic drug treatment can also occur from the defective or loss of mitochondria during mitotic arrest ${ }^{34}$. As such, impairment of mitochondrial $\beta$-oxidation promotes LD formation by diverting FA towards storage in $\operatorname{LDs}^{14,15}$.

The duration of mitotic arrest is known to be tightly linked to the extent of multinucleation and DNA damage following mitotic slippage ${ }^{5}$. We did not observe any significant increase in LD levels in cells treated with ZM447439, an Aurora kinase inhibitor that shortens the duration of mitotic arrest by overcoming the spindle assembly checkpoint. Blocking lipid biosynthesis by TOFA promoted early mitotic slippage, suggesting a role for lipids in ensuring proper mitotic progression. Support for this can be garnered from the finding that small interfering RNA-mediated depletion of lipid biosynthesis enzymes such as DGAT2 (a TAG synthesising enzyme) resulted in defective cell division, reminiscent of mitotic slippage $^{35}$. Incidentally, secreted factor(s) from tumourassociated macrophages (TAMs) have been found to suppress the cytotoxicity effects of antimitotic agents by promoting early mitotic slippage and cancer cell survival following paclitaxel treatment ${ }^{36}$. It will be of interest to investigate whether TAMs engender mitotic slippage via modulation of lipid biosynthetic pathways.

Several inflammatory signalling pathways have been proposed to be activated by multinucleated cells ${ }^{37}$. Our previous study had shown pro-tumorigenic inflammatory factors to be secreted by senescent cells post slippage ${ }^{8}$. Inhibition of autophagy abrogated the induction of senescence and its associated secretion of inflammatory factors. Although the inhibition of lipid biosynthesis did not affect the establishment of senescence post slippage, we observed an alteration in the secretory profile of cells. The inhibition of ACC and FASN by TOFA and C75 respectively differentially promoted opposing effects on the production of secretory factors post slippage and consequent tumour-promoting effects (Fig. 8). This disparity could result from the regulation of mitochondrial FA oxidation by the different lipid inhibitors used. FA oxidation is known to regulate the expression and secretion of cytokines in various cell types such as in oncogeneinduced senescent cells, tumour-associated macrophages and myeloid-derived suppressor cells ${ }^{38-41}$. We speculate and hypothesise that TOFA treatment decreases the level

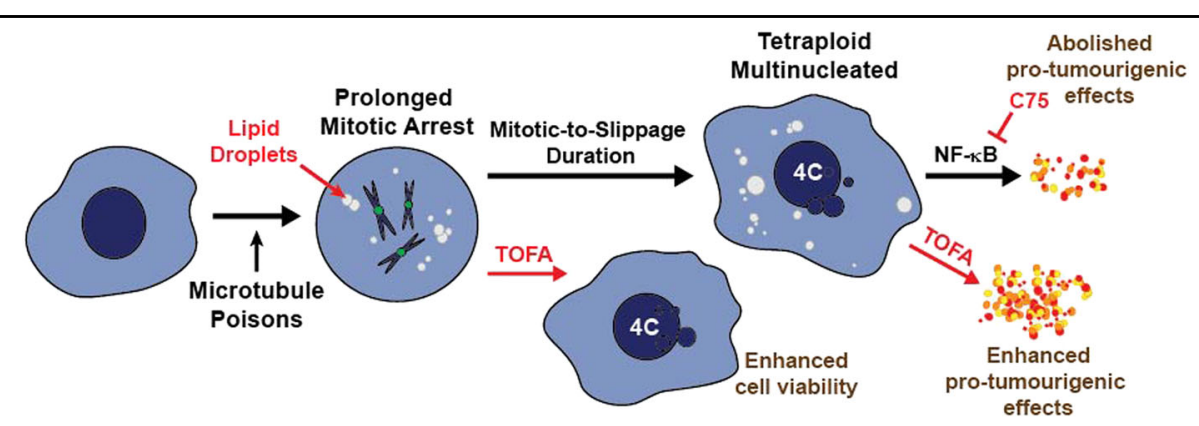

Fig. 8 Proposed model depicting the role of lipid biosynthesis in the regulation of cell fate upon antimitotic drug treatment. Upon treatment with antimitotic drugs, mitotic-arrested cells accumulate lipid droplets (LDs) that persist during and after mitotic slippage. Co-treatment with acetyl-COA carboxylase inhibitor TOFA and antimitotic drugs shorten the duration of mitotic arrest resulting in LD-depleted cells post slippage. Treatment of TOFA amplifies the secretion of tumour-promoting inflammatory factors and its associated effects, whereas treatment with C75, a fatty acid synthase inhibitor, shows an opposing phenomenon. Taken together, these indicate that there is differential regulation of the de novo fatty acid synthesis pathway in controlling phenotypic effects post slippage 
of malonyl-CoA, thereby preventing inhibition of FA oxidation as per scheme on Supplementary Fig. $3 \mathrm{a}^{42}$. On the contrary, inhibition of FASN by C75 may promote the accumulation of malonyl-CoA, thereby further suppressing FA oxidation and the subsequent production of protumourigenic factors ${ }^{43,44}$. Further work will need to be performed to confirm this hypothesis.

Alternatively, pro-tumourigenic factors could be altered by FA levels and saturation. It has been shown that fatty acids induce the expression and secretion of inflammatory cytokines such as IL-6, IL- 8 and IL- $1 \beta$ via activation of NF- $k B$ pathway in normal and cancer cells ${ }^{45-47}$. Indeed, our data revealed that $\mathrm{C} 75$ treatment suppressed the activation of NF- $\mathrm{kB}$ in post-slippage cells which supports the notion that increased FASN promotes the induction of post-slippage secretory factors. Another plausible inflammatory pathway involved could be via lipid mediators such as prostaglandin that is produced through conserved lipid biosynthesis pathways involving specific enzymes such as ACC and FASN. Collectively, our data suggest that lipid biosynthesis is involved in the modulation of pro-tumourigenic factors induced by cells post slippage.

Apart from the enhanced cell migration and invasion induced by post-slippage secretory factors, a distinct inflammatory response could also influence immune cell infiltration and the subsequent clearance of cells post slippage $^{4}$. Alternatively, the pro-tumourigenic cytokines secreted may also stimulate the polarisation of M1 macrophages to M2 TAMs that may further enhance the protumourigenic properties of neighbouring cells ${ }^{40}$. Indeed, paclitaxel treatment induces the infiltration of TAMs into mammary adenocarcinoma and suppresses antitumor Tcell response $^{28}$, which may invoke properties of senescence and SASP post slippage. More extensive work will be needed to determine the effect of antimitotic-induced pro-tumourigenic factors on immune cells present in the tumour microenvironment and whether lipid metabolism is involved in mediating these effects.

\section{Materials and methods}

\section{Cell culture and reagents}

Human osteosarcoma U2OS, colorectal carcinoma HCT116 and breast adenocarcinoma MDA-MB-231 cancer cell lines were purchased from American Type Culture Collection (ATCC). Cells were maintained in Dulbecco's modified Eagle's medium-high glucose (Gibco), 10\% FBS (GemCell) and 100 units/mL penicillin. Chemicals used were as follows: $100 \mathrm{ng} / \mu \mathrm{L}$ Nocodazole (N3000) (US Biological), $150 \mathrm{nM}$ Paclitaxel (Taxol) (P1792A) (US Biological), $3 \mathrm{mM}$ Thymidine (T5290) (Sigma), $100 \mu \mathrm{M}$ Monastrol (1305) and $2 \mu \mathrm{M}$ ZM447439 (2458) (TOCRIS). Lipid biosynthesis inhibitors used were as follows: Triascin C (ab141888) (Abcam), TOFA
(T6575), C75 (C5490) and Orlistat (O4139) (Sigma). For cell synchronisation, cells were subjected to $24 \mathrm{~h}$ of serum starvation (0\% FBS) followed by $3 \mathrm{mM}$ thymidine arrest (G1/S arrest). Cells were released from thymidine for $3 \mathrm{~h}$ before the addition of fresh media with respective drugs.

\section{Immunoblotting}

Whole-cell lysates were prepared in Pierce RIPA Buffer with Halt ${ }^{\mathrm{TM}}$ Protease \& Phosphatase Inhibitor Cocktail (Thermo Scientific). Protein concentration was measured using Bradford assay (Bio-Rad). An appropriate volume of Laemmli sample buffer was added to equal amounts of total proteins followed by boiling for $5 \mathrm{~min}$. Cells lysates were resolved by TRIS-glycine sodium dodecyl sulphate-polyacrylamide gel electrophoresis gels (BioRad), transferred onto nitrocellulose membranes and the membranes then incubated with primary antibodies overnight at $4{ }^{\circ} \mathrm{C}$. The immunoblots were subsequently washed and incubated with horseradish peroxidaseconjugated anti-mouse or -rabbit antibodies (Amersham Biosciences) for $1 \mathrm{~h}$ before detection by ChemiDoc MP system (Bio-Rad) using ECL substrate (Thermo Scientific). Antibodies used were: Abcam: Lamin B1 (ab65986); IкB $\alpha$ (ab7217), Nup153 (ab171074); Bethyl:BubR1 (A300386A); Cell Signaling Technology (CST): cleaved caspase3 (Asp 175) (9664S), cleaved PARP (Asp214) (9546), phospho-histone H3 (Ser10) (9701S), phospho-histone H2A.X (Ser139) (2577), phospho-NF-kB p65 (Ser536)

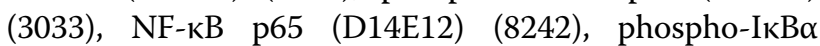
(Ser32/36) (9246), p21 Waf1/Cip1 (12D1) (\#2947); Santa Cruz: Actin (sc-47778), cyclin B1 (H-20) (sc-594), p53 (DO-1) (sc-126); Novus Biologicals: CXCL8/IL-8 (6217), IL-1 beta/IL-1F2 (8516).

\section{Oil Red $O$ staining}

A working concentration of Oil Red O solution was made by diluting a $3.5 \mathrm{mg} / \mathrm{mL}$ stock solution (O0625) (Sigma) (in 100\% isopropanol) with distilled water (6:4 ratio by volume). This solution was incubated at room temperature for $30 \mathrm{~min}$ and filtered through $0.2 \mu \mathrm{m} \mathrm{syr}-$ inge filter unit before use. Fixed cells were incubated in $60 \%$ isopropanol for $1 \mathrm{~min}$, dried at room temperature and incubated in Oil Red O staining solution for $1 \mathrm{~min}$. Cells were rinsed with distilled water two to five times and counterstained with Hoechst 33342 before mounting in ProLong Gold Antifade (P36935) (Life Technologies). Bright field images were captured using Nikon High Speed Live Cell Microscope (C11578-22C) with 40x objective.

\section{BODIPY 493/503 staining}

BODIPY 493/503 (D3922) (Thermo Scientific) was purchased from Life Technologies. Live cells were trypsinised and washed twice in phosphate-buffered saline 
(PBS) before incubating in $2 \mu \mathrm{g} / \mathrm{mL}$ BODIPY (in PBS) for $15 \mathrm{~min}$ at $37^{\circ} \mathrm{C}$. After staining, cells were washed twice in PBS and analysed on an Accuri C6 flow cytometer (BD Biosciences) under FL-1 channel. Data were analysed using FlowJo software.

\section{Fatty acid uptake assay}

Noc-treated cells were seeded at 50,000 cells $/ 100 \mu \mathrm{L}$ in each well of a 96-well plate. Cells were incubated in serum-free media at $37^{\circ} \mathrm{C}$ for $1 \mathrm{~h}$. For measurement of fatty acid uptake, the Free fatty acid uptake kit (Abam ab176768) used. Fatty acid dye-loading solution was then added to cells and fluorescence intensities measured at $20 \mathrm{~s}$ interval for $60 \mathrm{~min}$ with a fluorescence microplate reader (Biotek Cytation 5) at $\mathrm{Ex} / \mathrm{Em}=485 / 515 \mathrm{~nm}$ using a bottom read mode.

\section{Cell viability assay}

For relative cell viability, cells were seeded in 96-well plates and treated as per their respective duration. Cell viability was assessed using CellTiter $96^{\circledR}$ One Solution Proliferation Assays kit (G3580) (Promega) according to the manufacturer's instructions. For absolute cell viability, cells were seeded in $10 \mathrm{~cm}$ dishes and treated as per their respective duration. Cells were then trypsined, stained with $0.4 \%$ Trypan blue solution and counted using an automated cell counter $\left(\right.$ Luna $\left.^{\mathrm{mx}}\right)$. The number of viable cells $=$ Total number of cells - number of blue cells.

\section{Clonogenic survival assay}

U2OS cells were seeded at 7500 cells per well in 6-well plates. Cells were synchronised before the addition of respective drugs for $48 \mathrm{~h}$. The media were then removed, cells were washed twice and fresh media were added. Plates were incubated for 5 days until macroscopic colonies formed. For quantification of colonies, the cells were washed twice with ice-cold PBS and fixed for $10 \mathrm{~min}$ with $4 \%$ paraformaldehyde (PFA). Colonies were stained with a $0.4 \%$ crystal violet $/ 20 \%$ methanol solution for $10 \mathrm{~min}$. The cells were then washed with water to remove excess dye, and dried at room temperature overnight. Colonies were quantified by solubilisation in $100 \%$ methanol followed by spectrophotometric analysis at $570 \mathrm{~nm}$. The survival of treated cells was normalised relative to DMSO-treated cells. Statistical significance was determined by two-tailed Student's $t$-tests.

\section{Cellular ROS measurement}

Cells were trypsinised and washed twice in PBS before incubating in $20 \mu \mathrm{M}$ of 2', $7^{\prime}$-dichlorofluorescin diacetate (DCFDA) (ab113851) (Abcam) working solution for 25 min at $37^{\circ} \mathrm{C}$. After staining, cells were washed in PBS and analysed on an Accuri C6 flow cytometer (BD Biosciences) under FL-1 channel. Data were analysed using
FlowJo software. Fluorescence intensities were normalised against DMSO-treated cells.

\section{Preparation of conditioned media}

Synchronised cells were treated with respective drugs for $48 \mathrm{~h}$, followed by culture in drug-free media containing $1 \%$ FBS for additional $48 \mathrm{~h}$. After collection, culture media were centrifuged at $5000 \times g$, filtered through a $0.22 \mu \mathrm{m}$ pore filter (Pall Corporation) and mixed with media containing $40 \%$ FBS in a proportion of $3: 1$ to generate CM containing 10\% FBS.

\section{Human cytokine and chemokine array}

A total of $150 \mu \mathrm{L}$ of CM was collected per condition for the assay. Then, 64 cytokine/chemokine/growth factor biomarkers were quantified simultaneously by using a Discovery Assay ${ }^{\circledast}$ called the Human High Sensitivity TCell Discovery Array 64-Plex (Eve Technologies Corp, Calgary, AB, Canada). The multiplex assay was performed at Eve Technologies by using the Bio-Plex ${ }^{\text {TM }} 200$ system (Bio-Rad Laboratories, Inc., Hercules, CA, USA), and a Milliplex Human High Sensitivity T-Cell panel (Millipore, St. Charles, MO, USA) according to their protocol. The 64-plex consisted of EGF, Eotaxin, FGF-2, Flt-3 ligand, Fractalkine, G-CSF, GM-CSF, GRO, IFN- $\alpha 2$, IFN- $\gamma$, IL10, IL-12 (p40), IL-12 (p70), IL-13, IL-15, IL-17A, IL-1ra, IL-1 $\alpha$, IL-1 $\beta$, IL-2, IL-3, IL-4, IL-5, IL-6, IL-7, IL-8, IL-9, IP-10, MCP-1, MCP-3, MDC (CCL22), MIP-1 $\alpha$, MIP-1 $\beta$, PDGF-AA, PDGF-AB/BB, RANTES, TGF $\alpha$, TNF- $\alpha$, TNF$\beta$, VEGF, sCD40L, Eotaxin-2, MCP-2, BCA-1, MCP-4, I309, IL-16, TARC, 6CKine, Eotaxin-3, LIF, TPO, SCF, TSLP, IL-33, IL-20, IL-21, IL-23, TRAIL, CTACK, SDF-1a $+\mathrm{B}$, ENA-78, MIP-1d and IL-28A. The assay sensitivities of these markers range from 0.11 to $3.25 \mathrm{pg} / \mathrm{mL}$. The concentration of individual cytokines was determined based on the standard curve plotted from the determined concentration of a set standard and median fluorescent Intensity value.

\section{Cell migration and invasion assays}

For scratch wound migration assay, cells were seeded in 6-well plates to $90 \%$ confluency. A p200 pipet tip was used to create a scratch on the cell monolayer. Cells were incubated with respective $\mathrm{CM}$ and the wound closure rate was tracked. For the cell invasion assay, cells were incubated with $\mathrm{CM}$ and plated on the top surface of transwell filter chambers pre-coated or uncoated with Matrigel (BD Biosciences). After $24 \mathrm{~h}$ of incubation, noninvasive cells on the top chamber were removed by swapping the surface with cotton buds and washed twice with PBS. Invaded cells at the bottom chamber were fixed with $4 \%$ PFA and stained with $0.05 \%$ crystal violet. The percentage of invasive cells was quantified by cell counting. 
Senescence-associated $\beta$-galactosidase staining

Cells were stained using the SA- $\beta$-gal Staining Kit (\#9860) (CST) according to the manufacturer's instructions.

\section{Time-lapse imaging}

Cells were seeded in 6-well MatTek glass bottom dish at $4 \times 10^{5}$ per well in a volume of $1.5 \mathrm{~mL}$ culture media. Synchronised cells were then washed with $1 \times$ PBS and released in $1.5 \mathrm{~mL}$ fresh media before addition of the respective drugs. Imaging was performed using Nikon High Speed Live Cell Microscope (C11578-22C) with 40x objective, with image collected every $15 \mathrm{~min}$ using $25 \mathrm{~ms}$ and $50 \mathrm{~ms}$ exposures for differential interference contrast (DIC) and green fluorescent protein (GFP) channels respectively. Captured image sequences were viewed and analysed using MetaMorph software.

\section{Lipidomic analysis by mass spectrometry}

Six control cell samples (DMSO-treated) and six Nocodazole-treated cell samples $\left(3 \times 10^{6}\right.$ cells each) were freeze-dried to obtain the dry weight. Cells were extracted with a methanol/dichloromethane (DCM)/water (1:2:0.2) mixture. Water was subsequently added to obtain a lower phase DCM layer enriched with lipids. Dried lipid extracts were then reconstituted in $300 \mu \mathrm{L}$ of methanol. A quality control (QC) sample was created by combining $10 \mu \mathrm{l}$ aliquot from each extract. A dilution series of the QC sample and IS (internal standard) were first analysed to ensure the MS responses were linear over the concentration range of the analytes. The 12 lipid extracts and QC samples were diluted to appropriate concentration and then mixed with pre-determined concentration of IS which contains 14 deuterated standards representative for 14 lipid classes. The extracts and QC sample spiked with IS were then analysed in triplicates in randomised order. Data were acquired in full-scan mode in both positive and negative ionisation modes. A separate high-resolution mass spectrometry (HRMS)/MS data were acquired on the QC sample using "auto MS/MS" mode for the identification of the lipid species.

\footnotetext{
Acknowledgements

We thank members of the Crasta Lab for valuable comments. This research is supported by the National Research Foundation, Prime Minister's Office, Singapore under its NRF Fellowship Programme (NRF Award No. NRFNRFF2013-10), the Nanyang Assistant Professorship Grant, Nanyang Technological University Singapore and the Singapore Ministry of Education under its Singapore Ministry of Education Academic Research Fund Tier 1 (Grant No:2015-T1-002-046-01).

\section{Author details}

'Lee Kong Chian School of Medicine, Nanyang Technological University, Singapore, Singapore. ${ }^{2}$ Institute for Health Technologies, Interdisciplinary Graduate School, Nanyang Technological University, Singapore, Singapore. ${ }^{3}$ Institute of Molecular and Cell Biology, Agency for Science, Technology, and Research, Singapore, Singapore. ${ }^{4}$ Bioinformatics Institute, Agency for Science,
}

Technology, and Research, Singapore, Singapore. ${ }^{5}$ School of Biological Sciences, Nanyang Technological University, Singapore, Singapore

\section{Authors' contributions}

K.C. conceived and supervised the work done in this paper. A.W. and S.C. performed the experiments. L.K.Y. and Y.K. performed and analysed the mass spectrometry experiments. K.C. and A.W. analysed results and wrote the paper with help from the other authors.

\section{Conflict of interest}

The authors declare that they have no conflict of interest.

\section{Publisher's note}

Springer Nature remains neutral with regard to jurisdictional claims in published maps and institutional affiliations.

The online version of this article (https://doi.org/10.1038/s41420-018-0127-5) contains supplementary material, which is available to authorized users.

Received: 8 October 2018 Revised: 8 November 2018 Accepted: 12 November 2018

Published online: 27 November 2018

\section{References}

1. Chan, K. S., Koh, C. G. \& Li, H. Y. Mitosis-targeted anti-cancer therapies: where they stand. Cell Death Dis. 3, e411 (2012).

2. Jordan, M. A. \& Wilson, L. Microtubules as a target for anticancer drugs. Nat. Rev. Cancer 4, 253-265 (2004).

3. Brito, D. A. \& Rieder, C. L. Mitotic checkpoint slippage in humans occurs via cyclin B destruction in the presence of an active checkpoint. Curr. Biol. 16, 1194-1200 (2006)

4. Cheng, B. \& Crasta, K. Consequences of mitotic slippage for antimicrotubule drug therapy. Endocr. Relat. Cancer 24, T97-T106 (2017).

5. Zhu, Y., Zhou, Y. \& Shi, J. Post-slippage multinucleation renders cytotoxic variation in anti-mitotic drugs that target the microtubules or mitotic spindle. Cell Cycle 13, 1756-1764 (2014).

6. Ohashi, A. Different cell fates after mitotic slippage: from aneuploidy to polyploidy. Mol. Cell Oncol. 3, e1088503 (2016).

7. Coppe, J. P., Desprez, P. Y., Krtolica, A. \& Campisi, J. The senescence-associated secretory phenotype: the dark side of tumor suppression. Annu Rev. Pathol. $\mathbf{5}$, 99-118 (2010).

8. Jakhar, R. et al. Autophagy governs protumorigenic effects of mitotic slippageinduced senescence. Mol. Cancer Res. 16, 1625-1640 (2018).

9. Beloribi-Djefaflia, S., Vasseur, S. \& Guillaumond, F. Lipid metabolic reprogramming in cancer cells. Oncogenesis 5, e189 (2016).

10. Mashima, T., Seimiya, H. \& Tsuruo, T. De novo fatty-acid synthesis and related pathways as molecular targets for cancer therapy. Br. J. Cancer 100, 1369-1372 (2009).

11. Mehdizadeh, A. et al. Common chemotherapeutic agents modulate fatty acid distribution in human hepatocellular carcinoma and colorectal cancer cells. Bioimpacts 7, 31-39 (2017).

12. Li, N., Lizardo, D. Y. \& Atilla-Gokcumen, G. E. Specific triacylglycerols accumulate via increased lipogenesis during 5-FU-induced apoptosis. ACS Chem. Biol. 11, 2583-2587 (2016).

13. Cooper, W. A., Bartier, W. A., Rideout, D. C. \& Delikatny, E. J. 1H NMR visible lipids are induced by phosphonium salts and 5 -fluorouracil in human breast cancer cells. Magn. Reson. Med. 45, 1001-1010 (2001).

14. Boren, J. \& Brindle, K. M. Apoptosis-induced mitochondrial dysfunction causes cytoplasmic lipid droplet formation. Cell Death Differ. 19, 1561-1570 (2012).

15. Zirath, H. et al. MYC inhibition induces metabolic changes leading to accumulation of lipid droplets in tumor cells. Proc. Natl Acad. Sci. USA 110, 10258-10263 (2013).

16. Swanton, C. et al. Regulators of mitotic arrest and ceramide metabolism are determinants of sensitivity to paclitaxel and other chemotherapeutic drugs. Cancer Cell 11, 498-512 (2007). 
17. Flor, A. C., Wolfgeher, D., Wu, D. \& Kron, S. J. A signature of enhanced lipid metabolism, lipid peroxidation and aldehyde stress in therapy-induced senescence. Cell Death Discov. 3, 17075 (2017).

18. Zietkowski, D. et al. Comparison of NMR lipid profiles in mitotic arrest and apoptosis as indicators of paclitaxel resistance in cervical cell lines. Magn. Reson. Med. 68, 369-377 (2012).

19. Guo, Y., Cordes, K. R., Farese, R. V. Jr. \& Walther, T. C. Lipid droplets at a glance. J. Cell Sci. 122, 749-752 (2009).

20. Bensaad, K. et al. Fatty acid uptake and lipid storage induced by HIF-1alpha contribute to cell growth and survival after hypoxia-reoxygenation. Cell Rep. $\mathbf{9}$, 349-365 (2014).

21. Furuhashi, M. \& Hotamisligil, G. S. Fatty acid-binding proteins: role in metabolic diseases and potential as drug targets. Nat. Rev. Drug Discov. 7, 489-503 (2008).

22. Orth, J. D., Loewer, A., Lahav, G. \& Mitchison, T. J. Prolonged mitotic arrest triggers partial activation of apoptosis, resulting in DNA damage and p53 induction. Mol. Biol. Cell 23, 567-576 (2012).

23. Dikovskaya, D. et al. Mitotic stress is an integral part of the oncogene-induced senescence program that promotes multinucleation and cell cycle arrest. Cell Rep. 12, 1483-1496 (2015).

24. Gascoigne, K. E. \& Taylor, S. S. Cancer cells display profound intra- and interline variation following prolonged exposure to antimitotic drugs. Cancer Cell 14, 111-122 (2008).

25. Kuhajda, F. P. Fatty acid synthase and cancer: new application of an old pathway. Cancer Res 66, 5977-5980 (2006).

26. Hutti, J. E. et al. Oncogenic PI3K mutations lead to NF-kappaB-dependent cytokine expression following growth factor deprivation. Cancer Res. 72 3260-3269 (2012).

27. Collins, T. S., Lee, L. F. \& Ting, J. P. Paclitaxel up-regulates interleukin-8 synthesis in human lung carcinoma through an NF-kappaB- and AP-1-dependent mechanism. Cancer Immunol. Immunother. 49, 78-84 (2000).

28. DeNardo, D. G. et al. Leukocyte complexity predicts breast cancer survival and functionally regulates response to chemotherapy. Cancer Discov. 1, 54-67 (2011).

29. Peng, J. et al. Chemotherapy induces programmed cell death-ligand 1 overexpression via the nuclear factor-kappaB to foster an immunosuppressive tumor microenvironment in ovarian cancer. Cancer Res. 75, 5034-5045 (2015).

30. Deep, G. \& Schlaepfer, I. R. Aberrant lipid metabolism promotes prostate cancer: role in cell survival under hypoxia and extracellular vesicles biogenesis. Int. J. Mol. Sci. 17, pii: E1061 (2016).

31. Tabe, Y. et al. Bone marrow adipocytes facilitate fatty acid oxidation activating AMPK and a transcriptional network supporting survival of acute monocytic leukemia cells. Cancer Res. 77, 1453-1464 (2017).

32. Choi, J., Cha, Y. J. \& Koo, J. S. Adipocyte biology in breast cancer: From silent bystander to active facilitator. Prog. Lipid Res. 69, 11-20 (2018).
33. Nieman, K. M. et al. Adipocytes promote ovarian cancer metastasis and provide energy for rapid tumor growth. Nat. Med. 17, 1498-1503 (2011).

34. Domenech, E. et al. AMPK and PFKFB3 mediate glycolysis and survival in response to mitophagy during mitotic arrest. Nat. Cell Biol. 17, 1304-1316 (2015).

35. Atilla-Gokcumen, G. E. et al. Dividing cells regulate their lipid composition and localization. Cell 156, 428-439 (2014).

36. Olson, O. C., Kim, H., Quail, D. F., Foley, E. A. \& Joyce, J. A. Tumor-associated macrophages suppress the cytotoxic activity of antimitotic agents. Cell Rep. 19, 101-113 (2017).

37. Mitchison, T. J., Pineda, J., Shi, J. \& Florian, S. Is inflammatory micronucleation the key to a successful anti-mitotic cancer drug?. Open Biol. 7, pii: 170182 (2017).

38. Quijano, C. et al. Oncogene-induced senescence results in marked metabolic and bioenergetic alterations. Cell Cycle 11, 1383-1392 (2012).

39. Warfel, J. D. et al. Mitochondrial fat oxidation is essential for lipid-induced inflammation in skeletal muscle in mice. Sci. Rep. 6, 37941 (2016).

40. Zhang, Q. et al. Fatty acid oxidation contributes to IL-1 beta secretion in M2 macrophages and promotes macrophage-mediated tumor cell migration. Mol. Immunol. 94, 27-35 (2018).

41. Hossain, F. et al. Inhibition of fatty acid oxidation modulates immunosuppressive functions of myeloid-derived suppressor cells and enhances cancer therapies. Cancer Immunol. Res. 3, 1236-1247 (2015).

42. McCune, S. A. \& Harris, R. A. Mechanism responsible for 5-(tetradecyloxy)-2furoic acid inhibition of hepatic lipogenesis. J. Biol. Chem. 254, 10095-10101 (1979).

43. Pizer, E. S. et al. Malonyl-coenzyme-A is a potential mediator of cytotoxicity induced by fatty-acid synthase inhibition in human breast cancer cells and xenografts. Cancer Res. 60, 213-218 (2000).

44. Thupari, J. N., Pinn, M. L. \& Kuhajda, F. P. Fatty acid synthase inhibition in human breast cancer cells leads to malonyl-CoA-induced inhibition of fatty acid oxidation and cytotoxicity. Biochem Biophys. Res. Commun. 285, 217-223 (2001).

45. Hughes-Fulford, M., Li, C. F., Boonyaratanakornkit, J. \& Sayyah, S. Arachidonic acid activates phosphatidylinositol 3-kinase signaling and induces gene expression in prostate cancer. Cancer Res. 66, 1427-1433 (2006).

46. Jove, M., Planavila, A., Laguna, J. C. \& Vazquez-Carrera, M. Palmitate-induced interleukin 6 production is mediated by protein kinase $C$ and nuclear-factor kappaB activation and leads to glucose transporter 4 down-regulation in skeletal muscle cells. Endocrinology 146, 3087-3095 (2005).

47. Hu, M. B. et al. High-fat diet-induced adipokine and cytokine alterations promote the progression of prostate cancer in vivo and in vitro. Oncol. Lett. 15 1607-1615 (2018). 